\title{
Grit Blasting for Removal of Recast Layer from EDM Process on Inconel 718 Shaft: An Evaluation of Surface Integrity
}

\author{
Jonas Holmberg, Anders Wretland, and Johan Berglund
}

\author{
(Submitted May 3, 2016; in revised form September 13, 2016; published online October 26, 2016)
}

\begin{abstract}
The heat generated during EDM melts the work material and thereby allows large amounts to be removed, but an unfavorable surface of a recast layer (RCL) will also be created. This layer has entirely different properties compared to the bulk. Hence, it is of great interest to efficiently remove this layer and to verify that it has been removed. The main objective of this work has been to study the efficiency of grit blasting for removal of RCL on an EDM aero space shaft. Additionally, $x$-ray fluorescence (XRF) has been evaluated as a nondestructive measurement to determine RCL presence. The results show that the grit-blasting processing parameters have strong influence on the ability to remove RCL and at the same time introduce beneficial compressive stresses even after short exposure time. Longer exposure will remove the RCL from the surface but also increase the risk that a larger amount of the blasting medium will get stuck into the surface. This investigation shows that a short exposure time in combination with a short grit-blasting nozzle distance is the most preferable process setting. It was further found that handheld XRF equipment can be used as a nondestructive measurement in order to evaluate the amount of RCL present on an EDM surface. This was realized by analyzing the residual elements from the EDM wire.
\end{abstract}

Keywords aerospace, EDM, Inconel 718, residual stress, shot blasting, surface integrity, XRF

\section{Introduction}

In aerospace engine applications, structural parts often have large and complex geometries. Figure 1 presents an overview of an aircraft turbo fan engine. Many parts of the engine are made of heat-resistant nickel-based alloys such as Inconel 718. Machining of these alloys can be difficult and time-consuming due to their specific properties such as work hardening. The yellow circle indicates the type of component that this investigation is related to, namely a large shaft section in the fan structure.

A machining concept that sometimes can be very competitive for removal of large amounts of material quickly is electric discharge machining (EDM). This method utilizes the heat generated by the discharge from two electrodes, one of which is the part to be machined. The other electrode could either be a socalled die sink, which is a single tool that can generate holes and engravings, or a wire that can cut larger sections of the material.

The heat generated from the EDM wire (W-EDM) melts the material. Hence, the generated surface will contain an unfavorable recast layer (RCL). This recast layer can further be divided into three sub-layers according to Kumar (Ref 1), who

Jonas Holmberg, Swerea IVF AB - Manufacturing, Argongatan 30, Mölndal 431 22, Sweden and University West - Production Technology, Trollhättan 461 86, Sweden; Anders Wretland, GKN Aerospace Sweden AB, Trollhättan, Sweden; and Johan Berglund, Swerea IVF AB - Manufacturing, Argongatan 30, Mölndal 431 22, Sweden. Contact e-mail: jonas.holmberg@swerea.se. defines a spattered surface layer, a recast layer (white layer) and a heat-affected zone. The recast layer is generated by the melting and fast quenching of the material which alters the metallurgical state and becomes hard and brittle. The underlying heat-affected layer has not been subjected to melting, only heating (Ref 1). However, there are large differences in RCL depending on which type of work material that is processed by EDM. For steel, this layer becomes hard and brittle which cause microcracking of the surface layer (Ref 2, 3) with a typical thickness of the RCL of 10-20 $\mu \mathrm{m}$. According to the literature, the RCL of Inconel 718 does not show any microcracks but voids and hollow channels instead, which is believed to be due to the low carbon content of Inconel 718 (Ref 4). The thickness of the RCL for Inconel 718 is also thinner, typically less than $10 \mu \mathrm{m}$. Depending on process parameters, this layer could either appear as a discontinuous and non-uniform layer or a continuous thin layer (Ref 4).

There are several research papers investigating the surface integrity after EDM where the surface has been measured by means of microhardness, residual stresses, amount of microcracks and how the surface chemistry has been affected by the process. However, most of these papers concern conventional tool steels. Only a few concern surface integrity for more advanced materials, such as the nickel-based Inconel 718 . Newton et al. (Ref 5) showed an investigation of how the main W-EDM process parameters affect the characteristics of the recast layer formation when processing Inconel 718. It was shown that the RCL was typically 5-9 $\mu \mathrm{m}$ thick and that surface roughness mainly was affected by the EDM energy per spark. It was further shown that both $\mathrm{Cu}$ and $\mathrm{Zn}$ from the wire migrated from the wire electrode to the work piece.

In the study by Li et al. (Ref 4), the surface integrity of the RCL was investigated where a W-EDM surface of Inconel 718 was evaluated in terms of surface roughness, microhardness and chemical composition by energy-dispersive spectroscopy 
(EDS) analysis. The results showed a strong influence on the surface structure and roughness depending on discharge energy. It was also shown that the RCL had a much lower microhardness compared to the bulk indicating a thermal degradation regardless of discharge energy.

In order for a W-EDM processed surface to be used in the application addressed in the present paper the RCL first needs to be removed by some sort of post-process. The method proposed and evaluated in this paper is grit blasting.

Even though grit blasting is a well-established method no prior published results have been found where the influence of this process upon a material like Inconel 718 has been explored. There are, however, published results regarding shot peening where the influence of shot media, exposure time and distance to work piece has been investigated (Ref 6). Also to be mentioned is a work by Cai et al. (Ref 7) where an XRD method was presented to determine the precipitation kinetics of $\gamma$ " on shot peened Inconel 718 samples.

The only published study that is somewhat related to removal of RCL was presented by Wang et al. (Ref 8) who evaluated a method to remove the RCL by means of etching in combination with a mechanical grinding. The etching was performed with phosphoric acid coupled with hydrochloric acid in order to soften the RCL and thus allow it to be removed by grinding the surface.

Grit blasting could be a more environmentally acceptable alternative to etching for post-processing an EDM processed surface. However, in order to qualify grit blasting as a postprocess for EDM, the effects of the process parameters on the work piece with respect to surface integrity need to be fully understood, which has been the main objective with this investigation. A secondary objective was to investigate if chemical analysis of the surface can be used in order to qualify whether the surface is free from RCL or not.

\section{Experimental Set Up}

In this investigation, the surface integrity of EDM surfaces that were grit blasted, using different grit-blasting parameters, was evaluated (Fig. 2).

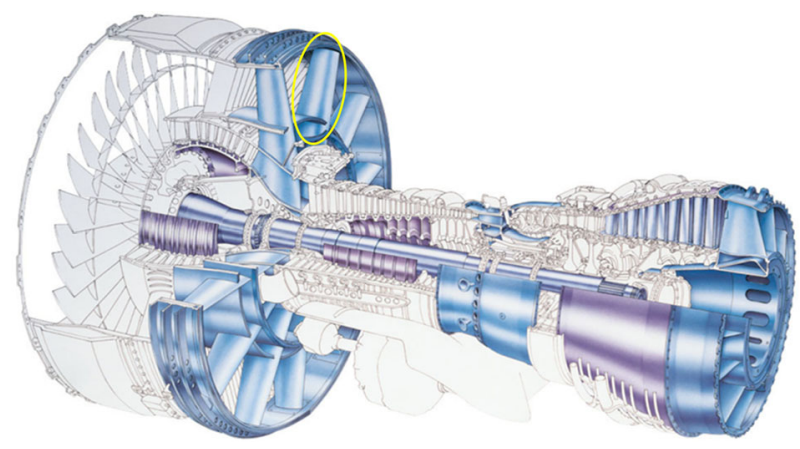

Fig. 1 Overview of an aircraft turbofan engine. Yellow circle indicates a typical component relevant for this investigation
The material of the shaft was Inconel 718 which has the chemical specification according to Table 1 .

The grit blast operation was performed in a robot cell where the EDM machined shaft was mounted on a turntable and the blast nozzle held by a robot in order to achieve a fully repeatable setup. The robot motion during the blast operation was single axis only, parallel to the shaft, whereas the attack of the stream of blast media was perpendicular to the shaft during all of the tests (Fig. 3). The grit medium used for these tests was aluminum oxide with a grit size of $60 \mu \mathrm{m}$ and a 4-bar pressure of the media.

The grit-blasting operation was performed with different process parameters in terms of number of passes (equivalent to exposure time) and nozzle distance (ND) which represents the distance between grit-blasting nozzle and the surface. Exposure time was varied between 2 and $40 \mathrm{~s}$ (exp) and the nozzle distance between 25 and $100 \mathrm{~mm}$.

Areas in between the test positions, dark shaded in Fig. 2, were masked during the grit blasting and were used as reference surfaces representing the state after EDM processing.

The EDM method used on this sample was a wire EDM, wire brand name bedra megacut ${ }^{\circledR}$ plus. This wire was composed of CuZn36 material with a so-called gamma-messing coating and had diameter of $0.25 \mathrm{~mm}$.

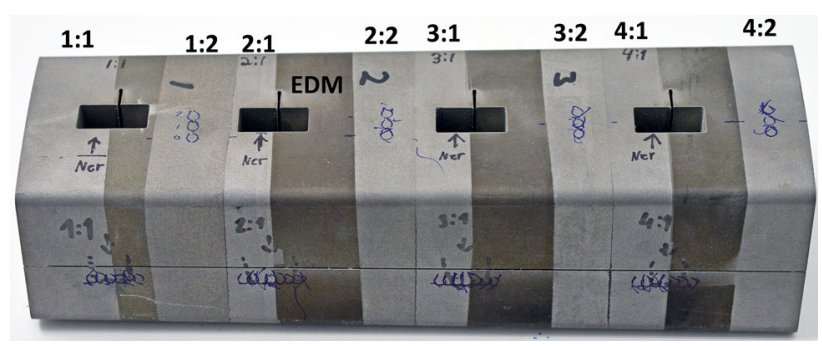

Fig. 2 Overview of the test sample, Inconel 718 shaft, showing the different grit-blasting positions
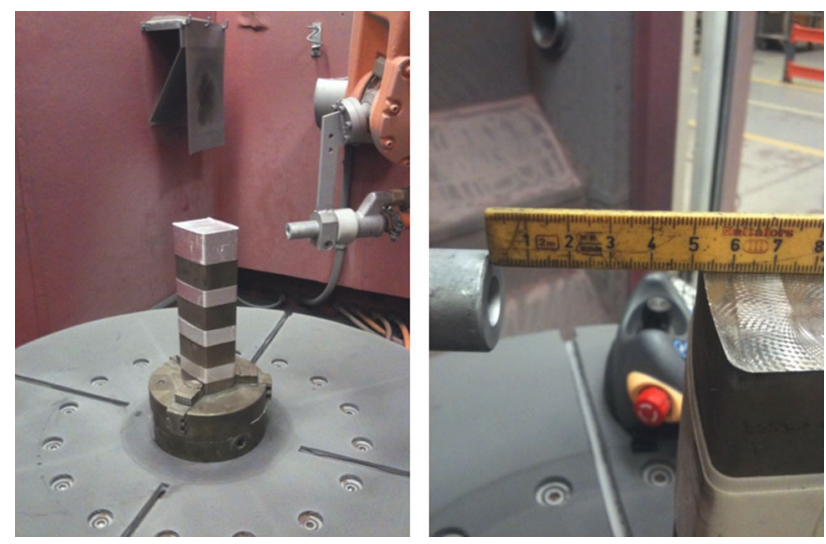

Fig. 3 Overview of the test setup. To the left the Inconel 718 shaft on the turn table just before the blast operation is to commence is shown. To the right the measured nozzle distance is shown

Table 1 Chemical specification of Inconel 718

\begin{tabular}{lccccccccccccc}
\hline $\mathrm{Ni}, \%$ & $\mathrm{Cr}, \%$ & $\mathrm{Fe}, \%$ & $\mathrm{Nb}, \%$ & $\mathrm{Mo}, \%$ & $\mathrm{Ti}, \%$ & $\mathrm{Al}, \%$ & $\mathrm{Co}, \%$ & $\mathrm{C}, \%$ & $\mathrm{Mn}, \%$ & $\mathrm{Si}, \%$ & $\mathrm{P}$ and S, \% & B, \% & $\mathrm{Cu}, \%$ \\
\hline $50-55$ & $17-21$ & Bal. & $4.75-5.5$ & $2.8-3.3$ & $0.65-1.15$ & $0.2-0.8$ & $<1.0$ & $<0.08$ & $<0.35$ & $<0.35$ & $<0.015$ & $<0.006$ & $<0.3$ \\
\hline
\end{tabular}




\section{Analysis and Evaluation}

The different analyses have been performed either to evaluate how the grit blasting has affected the material or to find a way to evaluate the RCL by means of nondestructive testing. Surface integrity of the samples has been evaluated using scanning electron microscope (SEM), energy-dispersive spectroscopy (EDS), residual stress measurement with $\mathrm{x}$-ray diffraction, chemical analysis of the surface with x-ray fluorescence (XRF) and evaluation of the RCL with optical microscopy.

The grit-blasting erosion depth was measured using the optical measurement and analysis equipment MikroCad from GFM. This equipment utilizes phase fringe projection consisting of parallel stripes which are projected onto the object surface, and their perspective deformation is recorded by a camera.

The grit-blasting erosion depth was measured over the different steps between EDM surface and the different blasting positions 1:1-4:2. Measurements were performed at a spot of $19 \times 19 \mathrm{~mm}$ for each position. The results were filtered and adjusted by form removal and performing a least square fit on the measured data. Finally, from the 1236 profiles across the step, an averaged profile was calculated and the step height was measured as the height between the levelled areas. The measurement height accuracy for this method is typically $\pm 1 \mu \mathrm{m}$. The different analysis steps for this evaluation are presented in Fig. 4-6.

Residual stress measurements were preformed with x-ray diffraction which is a well-established method to measure residual stresses at the surfaces as well as below the surface.
The measurements were performed with a Stresstech G2R XStress 3000 diffractometer equipped with a Mn X-ray tube $(\lambda: 0.21031 \mathrm{~nm})$. The modified $\sin ^{2} \psi$ method was used with 5 psi angles $\left(45^{\circ} \cdots-45^{\circ}\right)$ measuring the $151.88^{\circ}$ diffraction peak. The residual stress was calculated assuming elastic strain theory according to Hook's law using 196.6 MPa as Young's modulus and 0.29 in Poisson's ratio. Measurements of residual stress profiles were performed with layer removal where successive material removal is performed by electro polishing. The electro polishing was done with Struers Movipol and electrolyte A2.

A Jeol 6610LV SEM equipped with a Brucker XFlash 5010 EDS detector was used for chemical analysis of the surfaces. An EDS detector measures the electron interaction with the sample and generates energy spectrums that can be translated into chemical information. EDS is mainly used as a qualitative measurement to identify the chemical elements that are present on the sample surface. The chemical analysis of the surfaces was performed by EDS mapping which generates colored images of the distribution of the different chemical elements present on the surface.

The chemical content on the surface has also been measured with a handheld Thermo Scientific Niton XL3t GOLDD + xray florescence (XRF) equipment. This equipment utilizes the characteristic secondary $\mathrm{X}$-rays that emit from a sample surface which is exposed with high-energy $\mathrm{x}$-rays in order to determine the chemical content. This specific version of the equipment has an expanded range for detecting elements which includes $\mathrm{Mg}, \mathrm{Al}, \mathrm{Si}, \mathrm{P}$ and $\mathrm{S}$. The results from the chemical analysis with $\mathrm{XRF}$ are average values from three measurements at

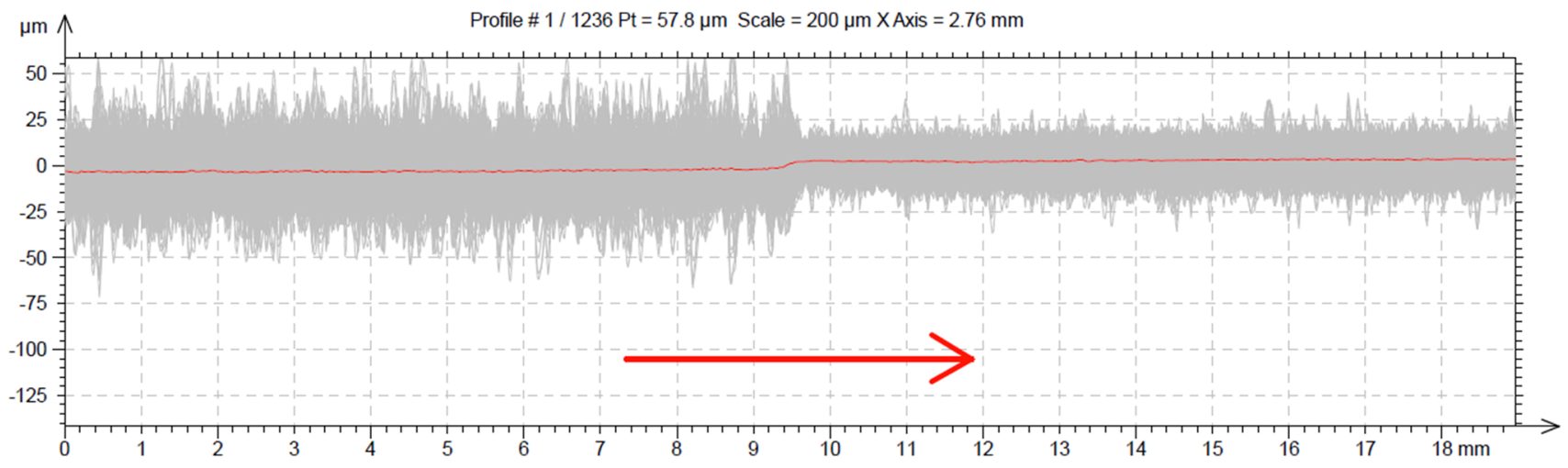

Fig. 4 Raw data from the erosion depth analysis

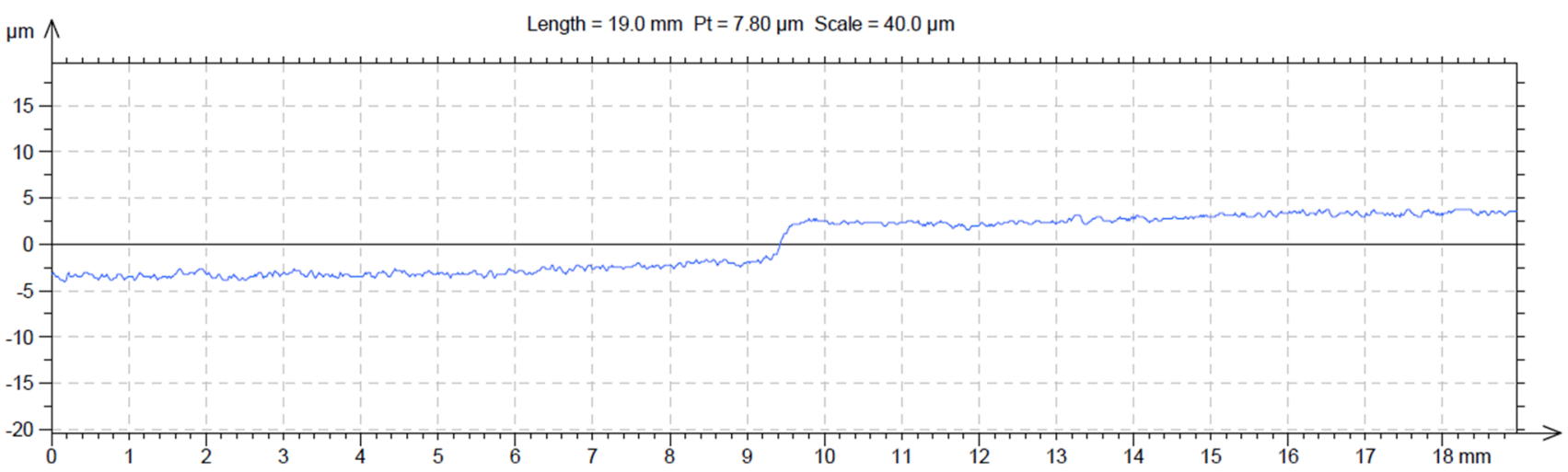

Fig. 5 Filtered and adjusted data 


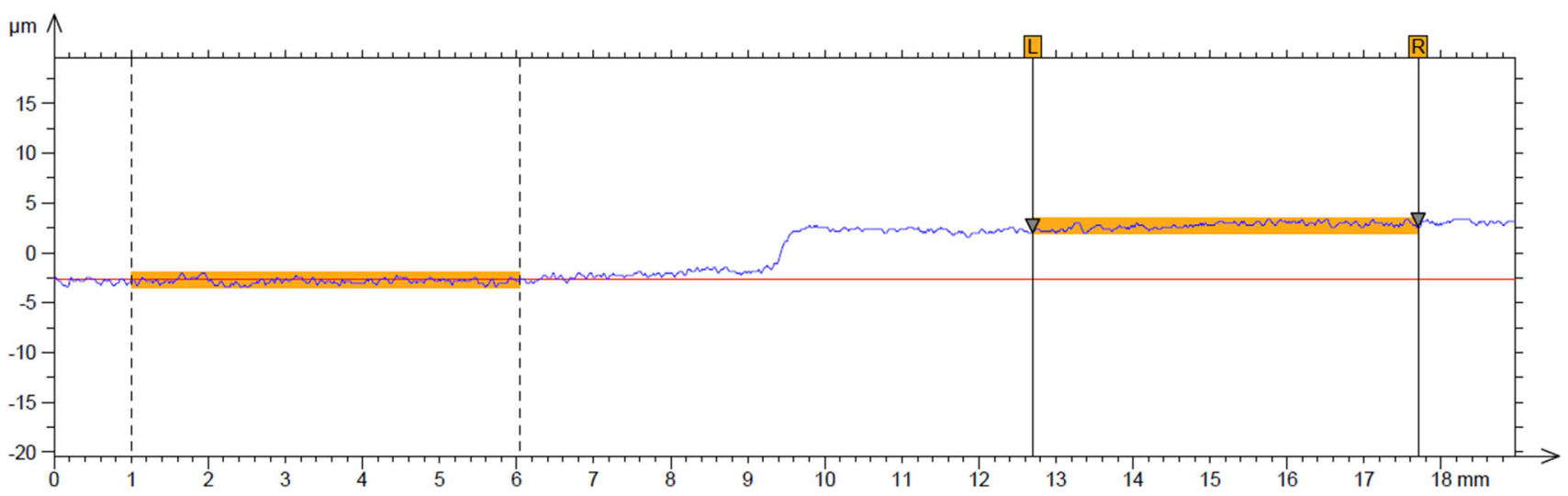

Fig. 6 Erosion depth calculated by use of two collimation lines

Table 2 Results from erosion depth measurements

\begin{tabular}{|c|c|c|c|c|c|}
\hline \multirow[b]{2}{*}{$\begin{array}{l}\text { Surface } \\
\text { marking }\end{array}$} & \multicolumn{2}{|c|}{ Shot-blasting parameters } & \multirow[b]{2}{*}{$\begin{array}{c}\text { Blasting exposure } \\
\text { time, } s\end{array}$} & \multirow[b]{2}{*}{$\begin{array}{l}\text { Material removal } \\
(\operatorname{Max}), \mu \mathrm{m}\end{array}$} & \multirow[b]{2}{*}{$\begin{array}{l}\text { Material removal } \\
(\text { Mean), } \mu \mathrm{m}\end{array}$} \\
\hline & $\begin{array}{l}\text { No. of } \\
\text { pass }\end{array}$ & $\begin{array}{l}\text { Nozzle distance, } \\
\text { mm }\end{array}$ & & & \\
\hline $1: 2$ & $\times 1$ pass & 100 & 2 & 1.0 & 0.1 \\
\hline $2: 2$ & & 100 & 4 & 3.6 & 2.7 \\
\hline $3: 2$ & & 100 & 8 & 7.6 & 6.9 \\
\hline $2: 1$ & $\times 5$ pass & 100 & 10 & 6.2 & 5.6 \\
\hline $3: 1$ & & 100 & 20 & 8.4 & 7.9 \\
\hline $4: 1$ & & 100 & 40 & 18.6 & 17.4 \\
\hline $4: 2$ & $\times 5$ pass & 25 & 40 & 12.8 & 12.2 \\
\hline $1: 1$ & $\times 5$ pass & 50 & 40 & 13.8 & 13.2 \\
\hline
\end{tabular}

different locations for each of the individual grit-blasting positions.

The microstructure was evaluated on polished and etched samples. The etching was performed with Kalling's solution in 30-60 s.

\section{Experimental Results}

\subsection{Erosion Depth and Surface Topography}

The results from the surface profile measurements show that the erosion from the grit blasting increases with increasing exposure time. It was also observed that blasting with longest nozzle distance and longest exposure time has resulted in the largest amount of erosion. Further, a masking effect could be seen close to the step which has resulted in a pit. Hence, the evaluation of step height was performed at a position 3-4 mm from the actual step. The results from the erosion depth measurements are summarized in Table 2.

\subsection{Residual Stresses}

The surface residual stress measurements were performed at different grit-blasting positions on the front side of the shaft according to Fig. 2. The results show that the blasting operation has a strong influence on the residual stresses according to Fig. 7. The stress state is predominantly compressive to a magnitude of $(-) 500-(-) 800 \mathrm{MPa}$ which is a great difference from the tensile stresses of approximately $200 \mathrm{MPa}$ which was measured for the reference EDM surfaces.

In Fig. 8, residual stress profiles for the grit-blasted surfaces with the same nozzle distance of $100 \mathrm{~mm}$ are presented. The residual stress profile measurements were performed with layer removal on the top side of the shaft according to Fig. 2. These results show that all profiles have the typical behavior from a grit-blasting process with high compressive stresses in the surface and a maximal compressive stress located 5-20 $\mu \mathrm{m}$ below the surface. The surface stresses, the maximal compressive stress and the penetration depth increase with increasing exposure time for most of the samples except for the 40 exp sample which does not follow this trend. Even the short $2 \mathrm{~s}$ grit-blasting exposure has resulted in a rather deep residual stress profile. A further increase in exposure time, comparing the 2 and 4 exp samples, shows further increase of $200 \mathrm{MPa}$ of the compressive stresses to a depth of $100 \mu \mathrm{m}$. The residual stress profiles for grit blasting more than $4 \mathrm{~s}$ are quite similar even though 10- and 20-s exposures seem to have a somewhat higher compressive stresses close to the surface compared to the other positions. The profile for 20-s exposure has the deepest impact according to Fig. 8. The reference EDM surface shows high tensile stresses deep inside the surface.

The residual stress profiles from blasting position 4:2, 1:1 and 4:1 show the influence of the nozzle distance and is presented in Fig. 9. These results indicate that the shortest ND, $25 \mathrm{~mm}$, generates the highest compressive stress both in terms of magnitude of the stresses and affected depth below the surface. Even at depths below $300 \mu \mathrm{m}$, this profile shows a compressive stress of $-400 \mathrm{MPa}$. The profiles for ND 50 and 


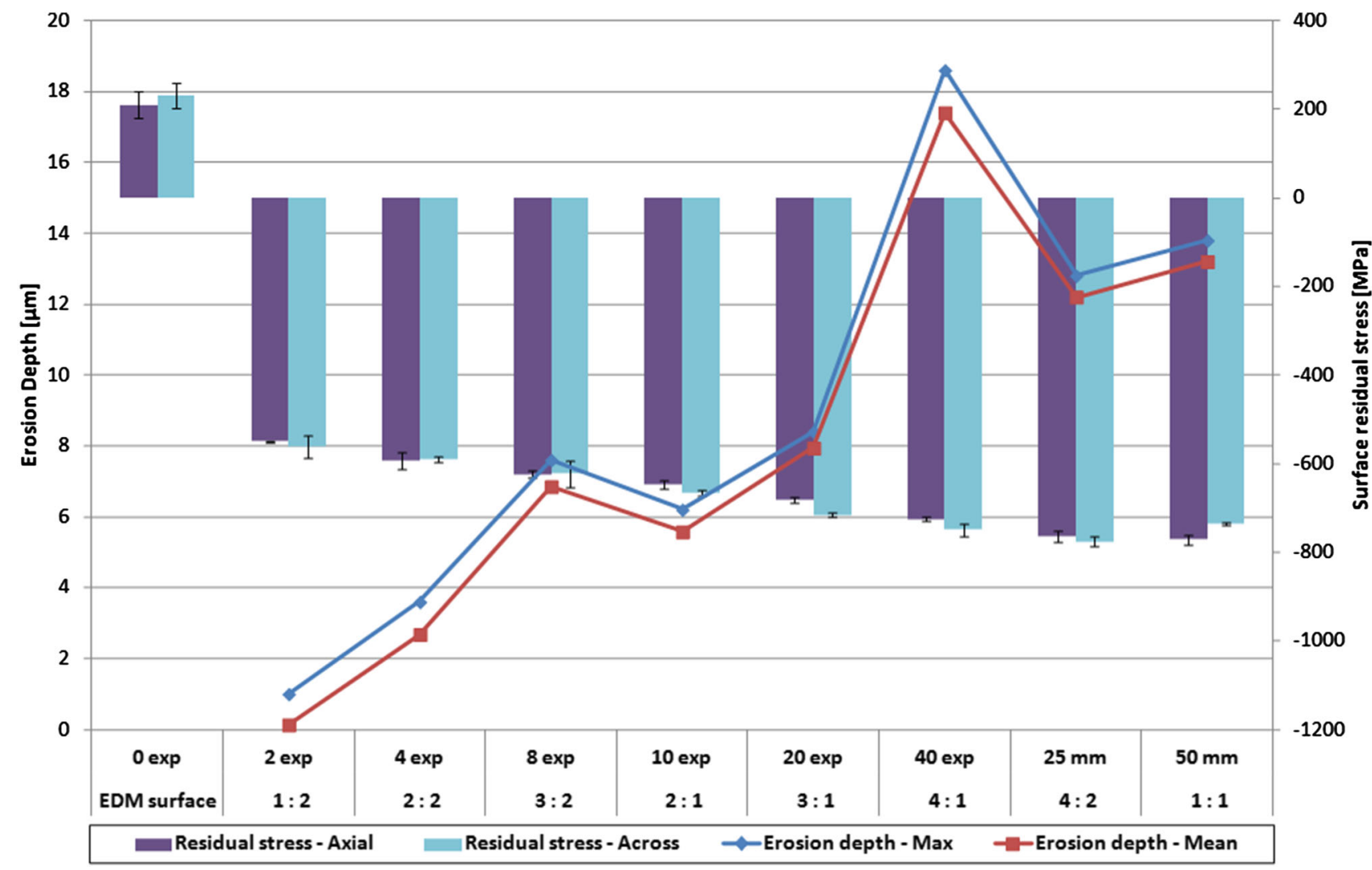

Fig. 7 Results of the erosion depth and surface residual stress measurements at the different blasting positions compared to the erosion measurement data. The error bars represent the standard deviations for the two residual stress measurements

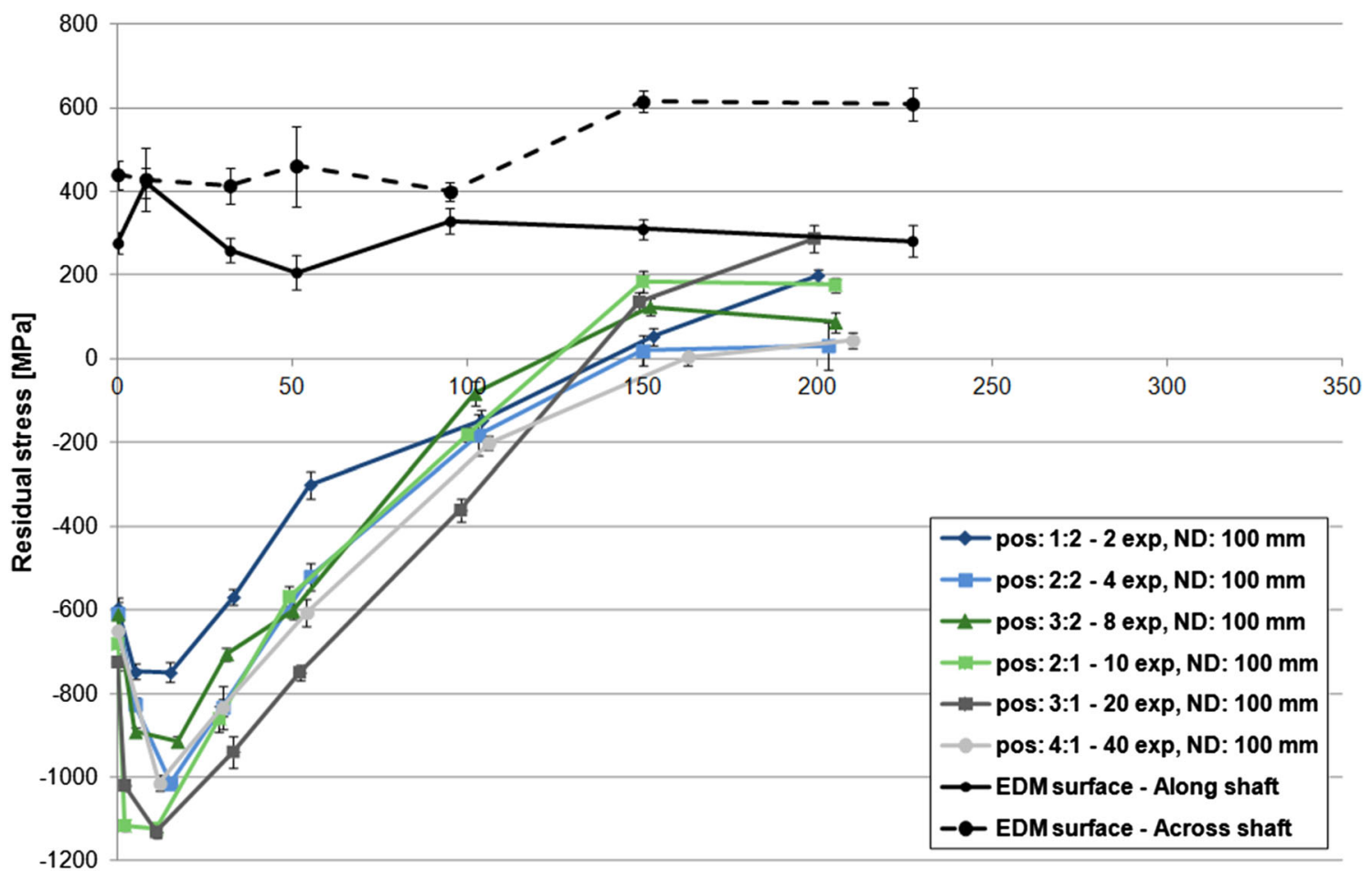

Depth below the surface $[\mu \mathrm{m}]$

Fig. 8 Results of the residual stress profile measurements at position 1:2, 2:2, 3:2 which differs in terms of exposure time in direction across the sample. The error bars represent the deviations of the diffraction peak curve fitting 


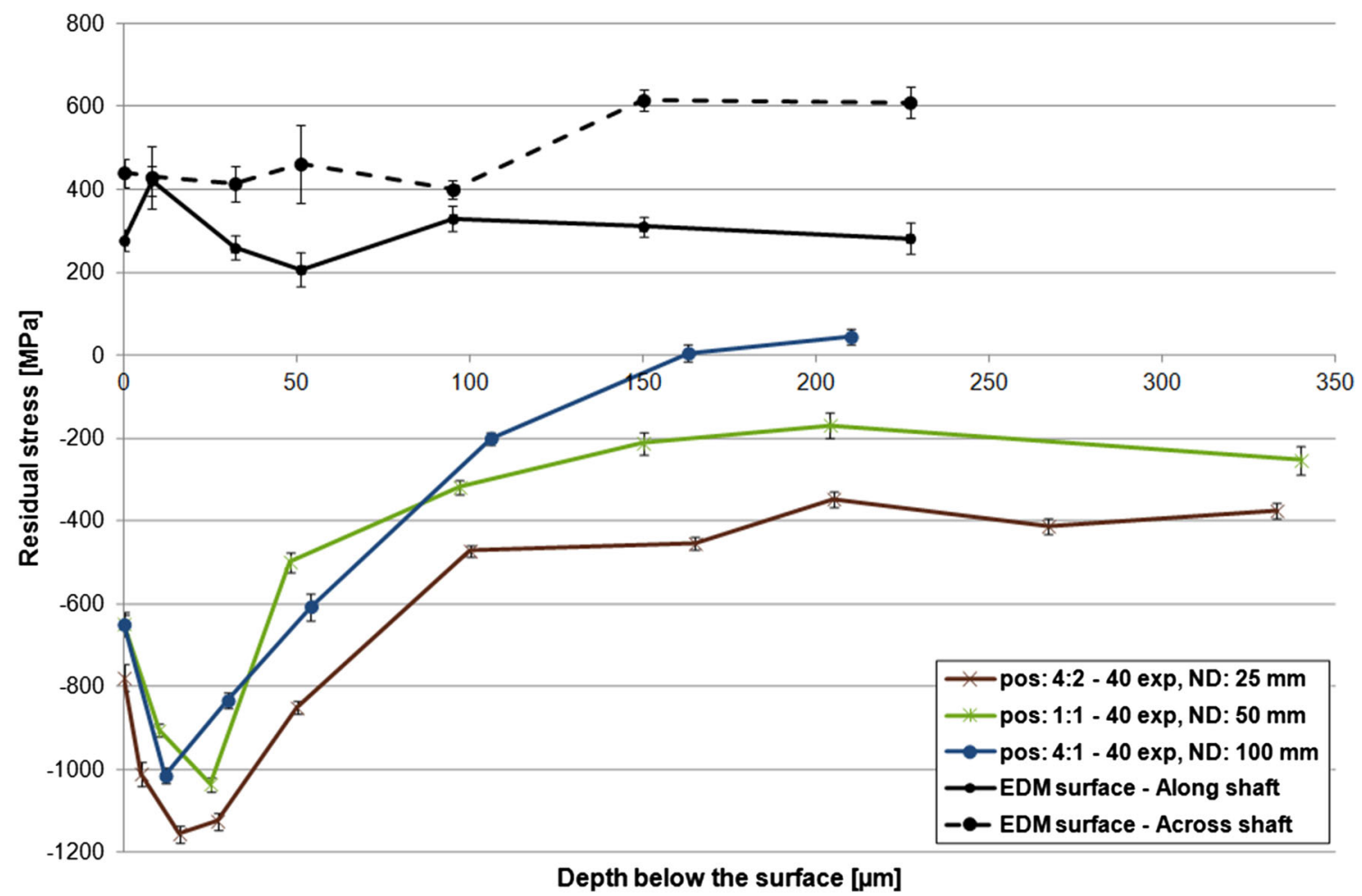

Fig. 9 Results of the residual stress profile measurements at position 4:2, 1:1 and 4:1 which differ in terms of nozzle distance (ND) in direction across the sample. The error bars represent the deviations of the diffraction peak curve fitting

\section{A: EDS map of nickel}

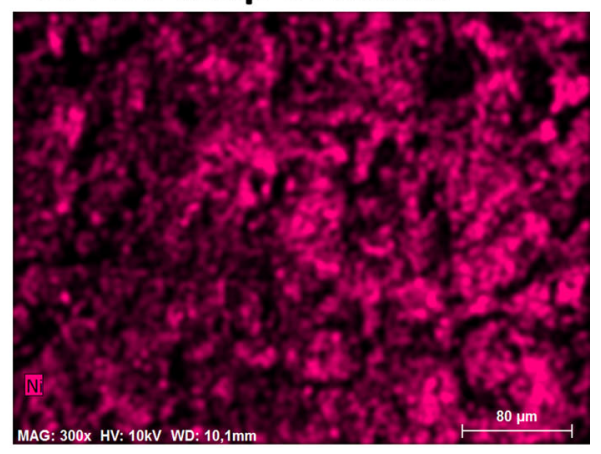

\section{B: EDS map of oxygen}

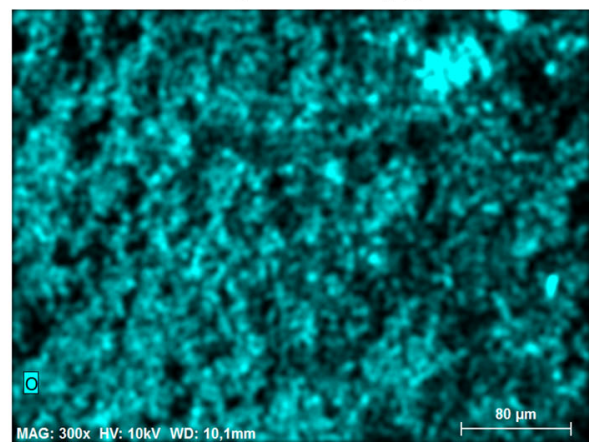

Fig. 10 EDS mapped surface in $300 \times$ magnification at the blasted step between masked and grit blast surface for sample 1:1 where (a) EDS map of nickel in purple color and (b) oxygen content in the green-colored surface 
$100 \mathrm{~mm}$ are quite similar to a depth of $100 \mu \mathrm{m}$. Below this depth ND $100 \mathrm{~mm}$ approaches a tensile stress, while the residual stress profile for ND $50 \mathrm{~mm}$ instead approaches a compressive stress of $-200 \mathrm{MPa}$ even at depths below $300 \mu \mathrm{m}$.

\subsection{Surface Chemistry: EDS Mapping}

The surfaces of the different blasting positions were further inspected by means of SEM and EDS mapping of the chemical content of the surface. These results imply that the EDM surface has a higher amount of oxygen and a lower amount of nickel, while the blasted surfaces show less surface oxides which could be seen in Fig. 10, whereas the more intense colored areas contain higher amounts of the elements oxygen and nickel.

In Fig. 11, the $\mathrm{Zn}$ content is illustrated in green color showing that the EDM surfaces have a much higher $\mathrm{Zn}$ content compared to the grit-blasted surfaces. It could further be seen that the $\mathrm{Zn}$ content decreases with increasing grit-blasting exposure time. Both zinc and copper are possible residue elements from the EDM wire that can indicate presence of RCL. These pictures also show that the grit-blasted positions with longer exposure times (3:2 and 4:2) have a lower amount of $\mathrm{Zn}$. It could further be observed that both sample $3: 2$ and $4: 2$ are similar, indicating that longer exposure times than 8 exp have less influence on the presence of $\mathrm{Zn}$.
Similar EDS maps were derived for copper, but comparing those images no clear difference could be observed.

\subsection{Surface Chemistry: XRF Surface Analysis}

The XRF results show that both $\mathrm{Zn}$ and $\mathrm{Cu}$ content decreases with increasing blasting exposure time and increased erosion depth according to Fig. 12. It can further be seen that the variation in the measurements of the EDM surface is large compared to the grit-blasted positions. Independently of the nozzle distance, long exposure times result in low contents of $\mathrm{Zn}$ and $\mathrm{Cu}$. Figure 13 shows how the measurements of $\mathrm{Ni}$ and $\mathrm{Cr}$ differ for the different positions. The error bars represent the standard deviation for the three measurements that were performed on different locations on the grit-blasted surface for each individual sample position. However, for the elements $\mathrm{Ni}$ and $\mathrm{Cr}$, the error bars are rather large for all sample positions which shows that the nickel and chromium content varies quite much in the analyzed surfaces. Therefore, it might be difficult to use the $\mathrm{Ni}$ and $\mathrm{Cr}$ content as an indicator of RCL removal. The nozzle distance shows some influence indicating a somewhat lower Ni content for the shorter ND.

\subsection{Evaluation of the RCL by Microscopy}

Evaluation of the recast layer was done on polished and etched cross sections of the shaft at positions at the steps between blasted and masked surface. The evaluation was done

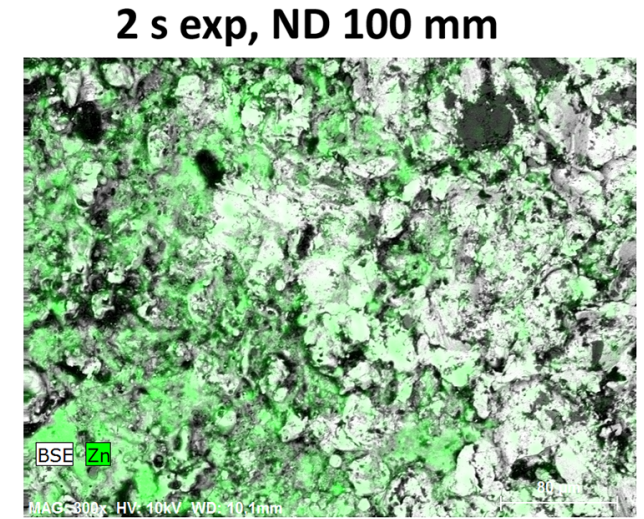

(a)

$8 \mathrm{~s} \exp$, ND $100 \mathrm{~mm}$

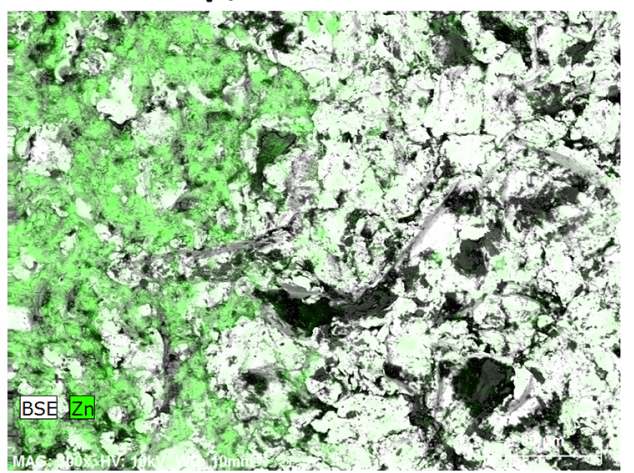

(c)
$4 \mathrm{~s} \exp$, ND $100 \mathrm{~mm}$

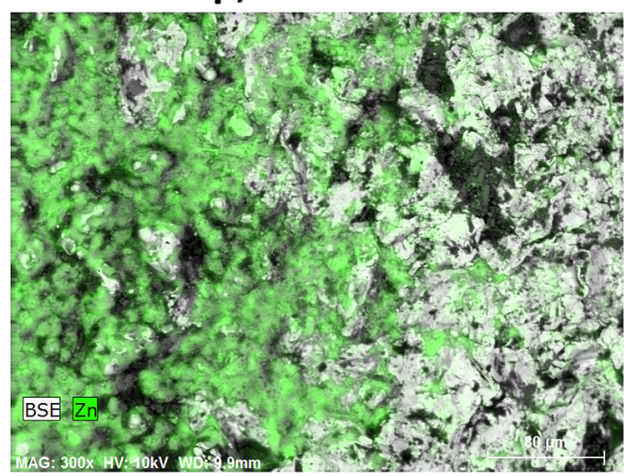

(b)

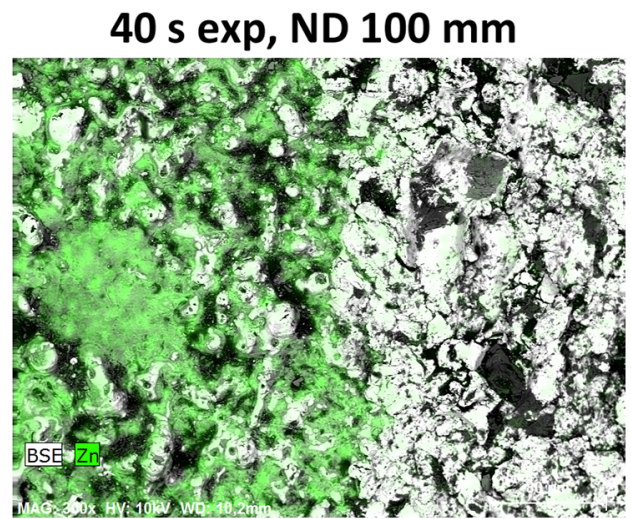

(d)

Fig. 11 EDS maps in 300x magnification of Zn content visualized in green at the blasting step between a masked and grit-blasted surfaces. (a) sample 1:2 with 2exp/ND $100 \mathrm{~mm}$, (b) sample 2:2 with 4 exp/ND $100 \mathrm{~mm}$, (c) sample 3:2 with 8 exp/ND $100 \mathrm{~mm}$ and (d) sample 4:2 with 40 $\exp / \mathrm{ND} 25 \mathrm{~mm}$ 


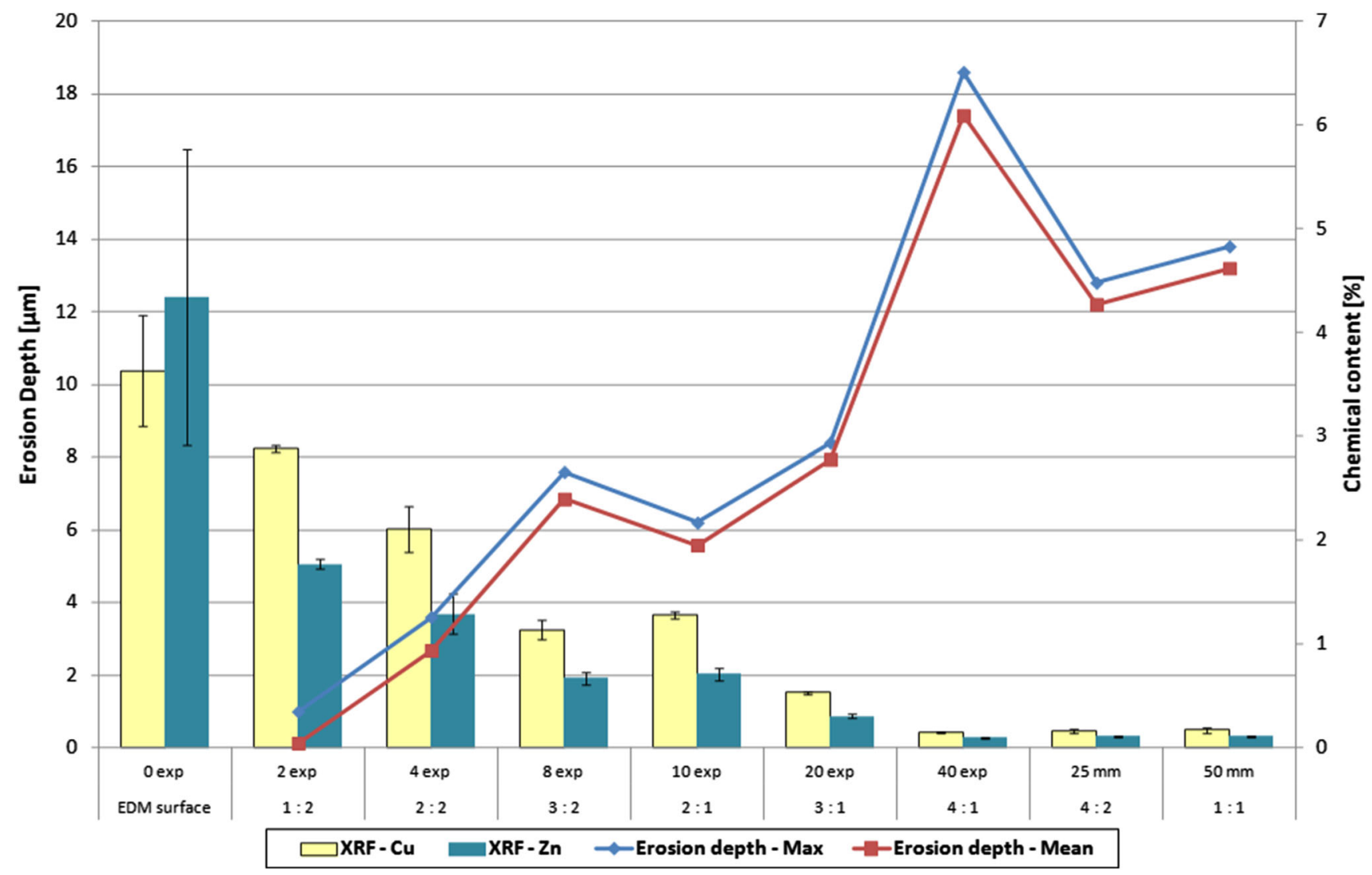

Fig. 12 Results of erosion depth and XRF chemical data measurements of $\mathrm{Zn}$ and $\mathrm{Cu}$ for the different blasting positions. The error bars represent the standard deviations for the three XRF measurements

at four positions for each individual blasting position, and representative micrographs in $\mathrm{x} 1250$ magnification are presented in Fig. 14. Recast layer could be detected on all EDM surfaces, but the amount differs a lot for the different locations. The RCL on the EDM surfaces is not continuous but instead appear as islands of RCL that typically only has an extension of less than $5 \mu \mathrm{m}$. The lower right images illustrate the appearance of the RCL which show a flake-like material that appears brighter in respect to the underlying bulk material.

RCL could only be observed for blasting position 1:2 and $3: 2$ where the later has only a minor amount embedded into the surface as a consequence of the grit blasting, see Fig. 14. All other samples show no traces of the RCL but differ instead regarding different amount of deformation of the outer surface. Some of the samples show relatively deep pits of erosion from the grit blasting.

\section{Discussion}

Although EDM is an efficient method for processing difficult materials to complex geometries the mechanical properties of the processed surfaces are often disadvantageous compared to other existing processing methods. Results presented by Navas where EDM was compared to grinding and hard turning of an AISI O1 tool steel showed that EDM was the most detrimental process (Ref 9). It has further been implied in several papers that the EDM of Inconel 718 generates a surface RCL that appears white when etching which is of different microstructure than the core ( $\operatorname{Ref} 4,5)$. This white layer is highly unwanted for functional and operating surfaces since the material properties might differ substantially compared to the bulk material. However, there has only been very limited work previously done regarding removal of this RCL with a method such as grit blasting even though the general opinion is that this is a well-established technique. In this study, the intension is to fill this gap of knowledge.

This study shows that grit blasting might be an efficient method for removal of the RCL and as an added value the surface will be plastically deformed into a compressive residual stress state. This compressive stress state is beneficial since it increases the fatigue strength of the material (Ref 10, 11).

The results reported in this paper show that the grit-blasting process has a strong influence on the surface characteristics for all of the investigated sample surfaces, even after a very short grit-blasting exposure time. The investigated surface topography, degree of deformation in microstructure and residual stress state have significantly been affected by the grit-blasting process. The results show that a long exposure time will remove the RCL from the surface, but this will also increase the risk that a larger amount of the blasting medium will get stuck into the blasted surface.

The typical residual stress profile from the related postprocess shot peening is a rather high compressive surface stress state that increases somewhat more a few micrometers below the surface. This is an effect of the Hertz's compression which, due to the impact, has a maximal plastic deformation that occurs below the surface (Ref 12). Typically, such results have been reported for different types of steel in the literature (Ref 


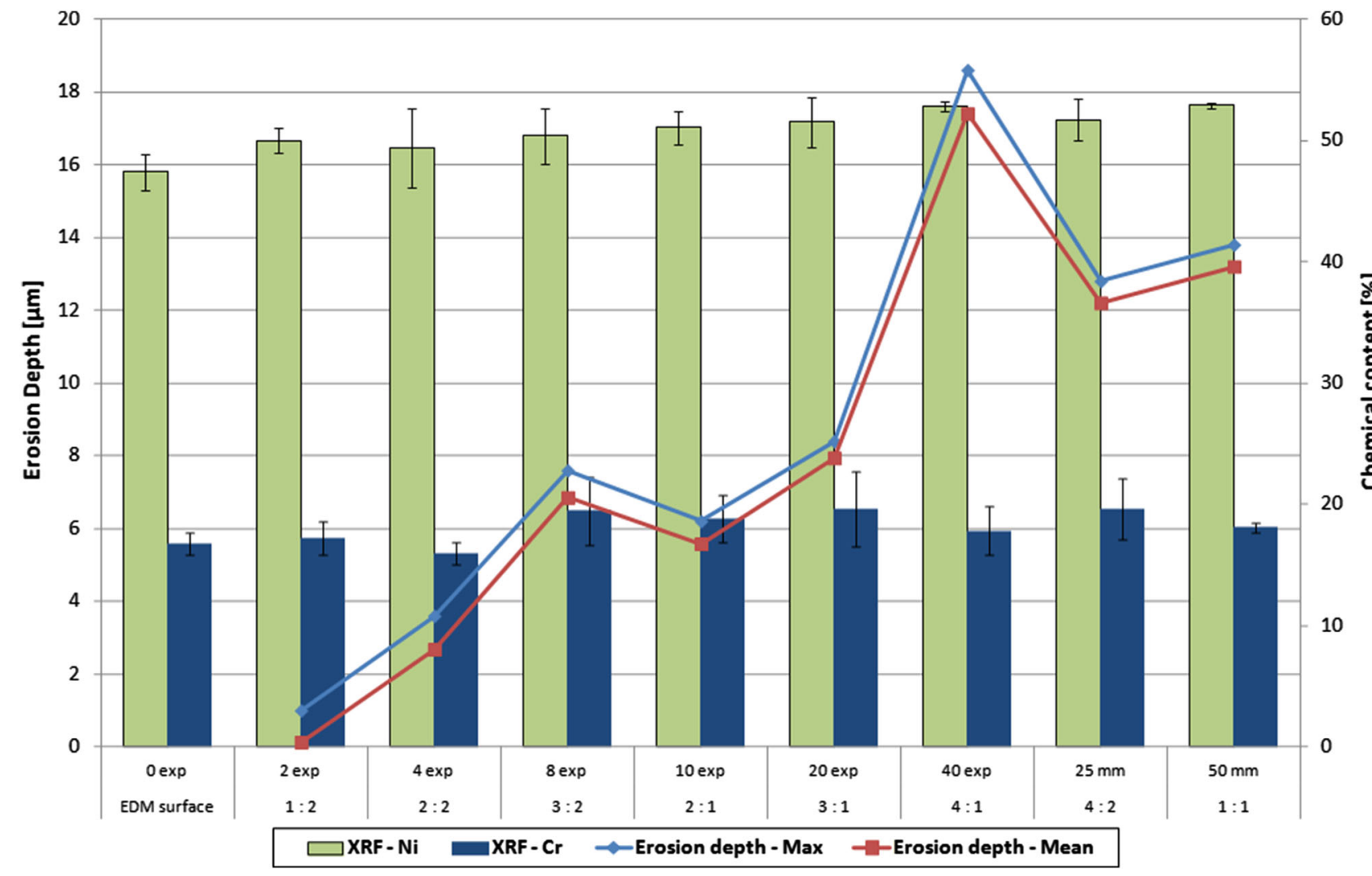

Fig. 13 Results of erosion depth and XRF chemical data measurements for Ni for the different blasting positions. The error bars represent the standard deviations for the three XRF measurements

11-14). In contrast, the residual stress measurements for the investigated grit-blasted Inconel shaft show an expected profile, but these profiles have a much higher maximal stress magnitude and a much lower penetration depth compared to other results, such as the results reported by Cai et al. (Ref 7) where the same super alloy was studied.

From these results, it could further be concluded that the exposure time has less influence and for longer exposure times than $4 \mathrm{~s}$ only minor increase in the compressive stresses could be observed. Though, for the shortest exposure time, $2 \mathrm{~s}$, a relatively high compressive stress has been generated in the surface. There is, however, an irregular trend to be observed where 10- and 20-s exposures show a much higher compressive residual stress just below the surface compared to $40-\mathrm{s}$ exposure. The reason for this need to be further investigated with a more thorough analysis of the microstructure.

The residual stress measurements further show that the nozzle distance has a somewhat stronger influence on both de maximal compressive stress and the penetration depth. For both the sample with short nozzle distance of $25 \mathrm{~mm}$ and the sample grit blasted with $50 \mathrm{~mm} \mathrm{ND}$, the residual stress state in the sample approaches a compressive residual stress compared to the profile for the reference EDM position which instead approaches a tensile stress deeper inside the sample. The relative high tensile stress state measured for the EDM position is surprisingly high, and it is believed to be a result of the cooling during the casting of the shaft rather than an effect of the EDM process which is only affecting the outermost surface.

The examination of the microstructure revealed that the degree of deformation also increased with decreased nozzle distance. This might also be an argument to use a shorter nozzle distance in order to retain maximal compressive stress in the surface. These analyses show how important it is to evaluate the surface characteristics when optimizing a RCL removal process. Both the residual stress state and the degree of deformation will have great influence on mechanical properties of the final surface. On the one hand, a high and rather deep compressive residual stress is preferable from a fatigue resistance perspective, but on the other hand a short nozzle distance will increase the deformation and risk of embedding unwanted grit-blasting medium particles in the surface. The investigation of the microstructure concludes that a rather short exposure time, $4 \mathrm{~s}$, generates a surface that appears to be free from RCL in light optical microscopy.

Additionally, it was found that one possible way of evaluating presence of RCL could be by performing chemical surface analysis using EDS. The analysis showed that there exist traces of the EDM wire electrode by means of zinc and copper in the EDM surface, which also was reported by Williams and Rajurkar (Ref 15). EDS mapping of the interface between the EDM surface and grit-blasted surface illustrates very well that the amount of primarily $\mathrm{Zn}$ differs, but difference in $\mathrm{O}$ and $\mathrm{Al}$ could also be detected. The difference was especially significant when comparing the EDM surface with the sample that was grit blasted with longer exposure times. Further, unwanted grit-blasting particles were detected in the surface. This could be seen as the darker areas in the images in Fig. 11. These images show that all blasted surfaces contain some amount of the $\mathrm{Al}$ particles that got stuck into the surface during the blasting operation, but increased exposure time will probably increase the amount of particles that get stuck in the surface. EDS analysis in SEM showed that it was possible to 

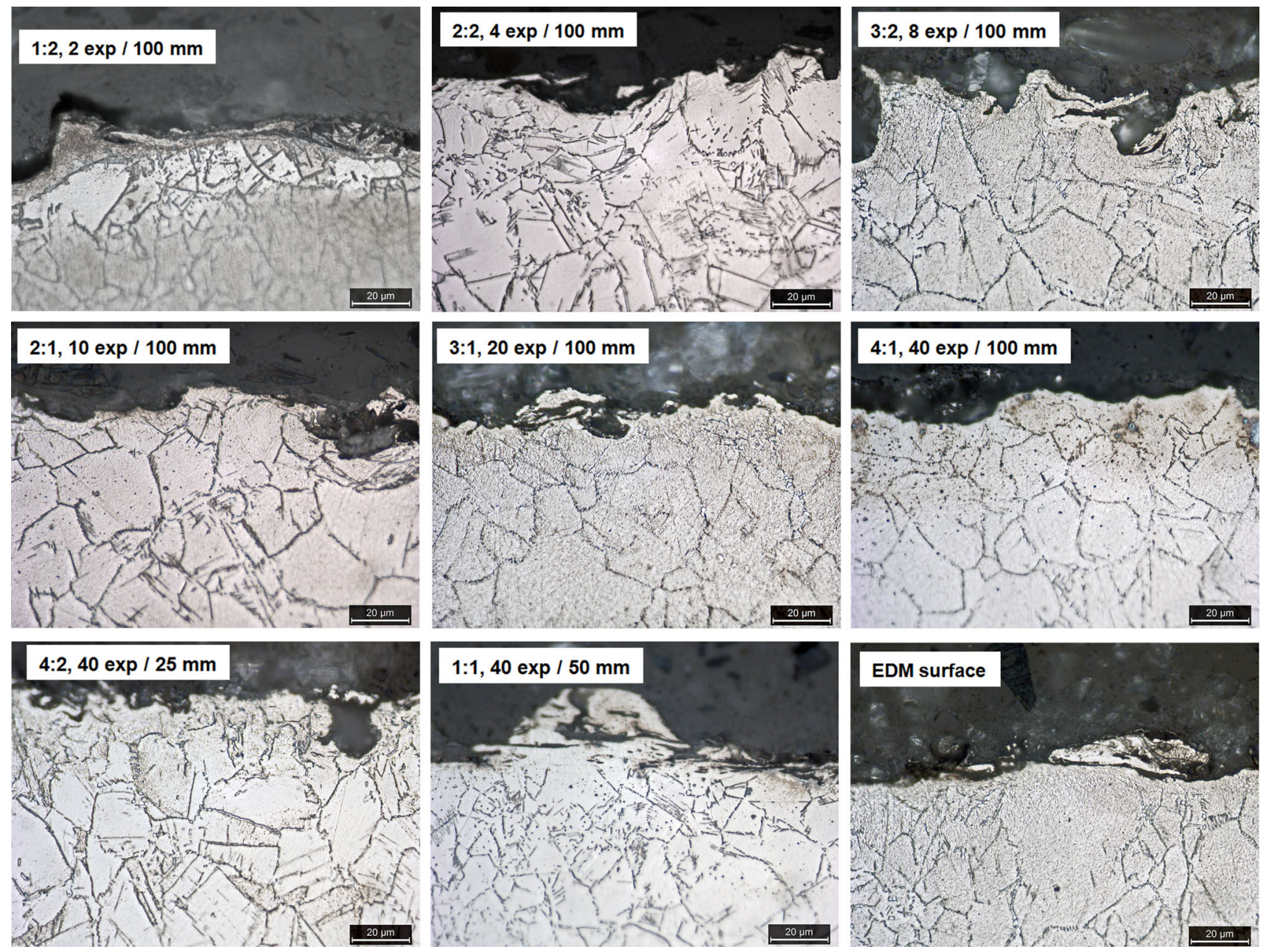

Fig. 14 Illustrative micrographs at 1250x magnification showing the microstructure of the grit-blasted surface for different grit-blasting parameters

detect a difference, but this type of analysis is time-consuming and not suitable if large surfaces are to be evaluated.

A nondestructive alternative to EDS was evaluated by means of hand held XRF equipment which has the advantage of measuring directly on the surface and without necessitating a vacuum environment. This equipment was selected to evaluate the surface chemistry since it is fast, nondestructive and is a mobile equipment that could be used for in-line process control. However, XRF does have some detection limitations regarding lighter elements due to both instrumentation and the low X-ray yields from lighter elements. Therefore, it is often difficult to quantify lighter elements than sodium. Even though the material Inconel 718 contains several alloying elements according to Table 1 it is only carbon and boron that cannot be quantified by the XRF measurement. All other elements can be quantified with good accuracy. Both carbon and boron represent very low amounts in the chemical composition of Inconel 718. Hence, the error of not detecting these elements will only have a marginal impact on the final quantitative results.

The selected strategy regarding how to evaluate the RCL with the XRF equipment is to analyze the surface regarding the copper and zinc content. The results showed that it is possible to detect and quantify the presence of residue from the EDM wire, even though the amounts are fairly low. The $\mathrm{Cu}$ and $\mathrm{Zn}$ content in the EDM surface is approximately $3.6 \pm 0.5$ and $4.3 \pm 1.4 \%$, respectively. While for a grit-blasted surface, these amounts drop to $2.9 \pm 0.03 \% \mathrm{Cu}$ and $1.8 \pm 0.05 \% \mathrm{Zn}$ after only $2 \mathrm{~s}$ exposure time. These amounts decrease even further with increased exposure time, and the longest exposure times show the lowest amount of these residues, indicating that the RCL is almost completely removed. In addition, the results show that the variation of the measured amounts also decreases, respectively. This indicates that XRF can be a suitable method to be used since it is fast and seems to be a reliable measurement in order to determine whether the surface is free from RCL or not.

\section{Conclusions}

- The results show that grit blasting is a possible method to be used in order to remove RCL after EDM processing. The grit-blasting parameters blasting time and nozzle distance have great influence on the resulting surface integrity generating a RCL-free surface with high compressive residual stresses after exposure times greater than $4 \mathrm{~s}$.

- Grit-blasting time only has a minor influence on the residual stress profile for exposure times longer than $4 \mathrm{~s}$. 
- Penetration depth and amplitude of the compressive stresses increase with shorter nozzle distance.

- Residue of the EDM wire has been detected on the EDM surface that could be used in order to determine presence of RCL.

- XRF measurements of the surface can be used as a nondestructive method for evaluation of whether RCL is still present on the sample surface after grit blasting.

\section{Acknowledgments}

The results presented in this paper are part of the research project G5Demo-2 and financed by VINNOVA, the Swedish government agency within Ministry of Enterprise. Special thanks to GKN for supplying test materials, information, overview image of the engine structure and expertise in the involved processes. The authors also would like to acknowledge the KK-foundation and the SiCoMap research school.

\section{Open Access}

This article is distributed under the terms of the Creative Commons Attribution 4.0 International License (http://creativecommons.org/ licenses/by/4.0/), which permits unrestricted use, distribution, and reproduction in any medium, provided you give appropriate credit to the original author(s) and the source, provide a link to the Creative Commons license, and indicate if changes were made.

\section{References}

1. S. Kumar, R. Singh, T.P. Singh, and B.L. Sethi, Surface Modification by Electrical Discharge Machining: A Review, J. Mater. Process. Technol., 2009, 209, p 3675-3687
2. L.C. Lee, L.C. Lim, and Y.S. Wong, Crack Susceptibility of ElectroDischarge Machined Surfaces, J. Mater. Process. Technol., 1992, 29, p 213-221

3. L.C. Lee, L.C. Lim, and Y.S. Wong, Towards Crack Minimisation of EDMed Surfaces, J. Mater. Process. Technol., 1992, 32, p 45-54

4. L. Li, Y.B. Guo, X.T. Wei, and W. Li, Surface Integrity Characteristics in Wire-EDM of Inconel 718 at Different Discharge Energy, Procedia CIRP, 2013, 6, p 220-225

5. T.R. Newton, S.N. Melkote, T.R. Watkins, R.M. Trejo, and L. Reister, Investigation of the Effect of Process Parameters on the Formation and Characteristics of Recast Layer in Wire-EDM of Inconel 718, Mater. Sci. Eng. A, 2009, 513-514, p 208-215

6. A. Sandá, V. García Navas, and O. Gonzalo, Surface State of Inconel 718 Ultrasonic Peened: Effect of Processing Time, Material and Quality of Shot Balls and Distance from Radiating Surface to Sample, Mater. Des., 2011, 32, p 2213-2220

7. D. Cai, P. Nie, J. Shan, W. Liu, M. Yao, and Y. Gao, Precipitation and Residual Stress Relaxation Kinetics in Shot-Peened Inconel 718, J. Mater. Eng. Perform., 2006, 15, p 614-617

8. C.-C. Wang, H.-M. Chow, L.-D. Yang, and C.-T. Lu, Recast Layer Removal After Electrical Discharge Machining Via Taguchi Analysis: A Feasibility Study, J. Mater. Process. Technol., 2009, 209, p 4134 4140

9. V. García Navas, I. Ferreres, J.A. Marañón, C. Garcia-Rosales, and J. Gil Sevillano, Electro-Discharge Machining (EDM) Versus Hard Turning and Grinding-Comparison of Residual Stresses and Surface Integrity Generated in ASIS O1 Toll Steel, J. Mater. Process. Technol., 2008, 195, p 186-194

10. Almen, J., Shot blasting to increase fatigue resistance, SAE Technical Paper, 51(7), 1943

11. S. Tekeli, Enhancement of fatigue strength of SAE 9245 steel by shot peening, Mater. Lett., 2002, 57(3), p 604-608

12. G. Totten, M. Howes, and T. Inoue, Handbook of Residual Stress and Deformation, ASM International, Almere, 2002, p 345-358

13. S. Bagherifad and M. Guagiliano, Fatigue of a Low-Alloyed Steel with Nanostructured Surface Obtained by Severe Shot Peening, Eng. Fract. Mech., 2012, 81, p 56-68

14. K. Dalaei and B. Karlsson, Influence of Shot Peening on Fatigue Durability of Normalized Steel Subjected to Variable Amplitude Loading, 2012, 38, p 75-83

15. R.E. Williams and K.P. Rajurkar, Study of Wire Electrical Discharge Machined Surface Characteristics, J. Mater. Process. Technol., 1991, 28, p 127-138 